\title{
Bulk Antisymmetric tensor fields coupled to a dilaton in a Randall-Sundrum model
}

\author{
G. Alencar ${ }^{1}$, M. O. Tahim ${ }^{1}$, R. R. Landim² , C. R. Muniz ${ }^{3}$ and R. N. Costa Filho ${ }^{2,4}$ \\ 1 Universidade Estadual do Ceará, Faculdade de Educação, \\ Ciências e Letras do Sertão Central, Quixadá,Ceará, Brazil. \\ ${ }^{2}$ Departamento de Física, Universidade Federal do Ceará, \\ Caixa Postal 6030, Campus do Pici, 60455-760, Fortaleza, Ceará, Brazil. \\ ${ }^{3}$ Universidade Estadual do Ceará, Faculdade de Educação, \\ Ciências e Letras de Iguatu, Rua Deocleciano Lima Verde, s/n Iguatu,Ceará, Brazil. \\ ${ }^{4}$ Department of Physics and Astronomy,University of Western Ontario London, Ontario, Canada N6A $3 K^{r}$.
}

(Dated: June 16, 2018)

\begin{abstract}
A string-inspired 3-form-dilaton-gravity model is studied in a Randall-Sundrum brane world scenario. As expected, the rank-3 antisymmetric field is exponentially suppressed. For each mass level, the mass spectrum is bigger than the one for the Kalb-Ramond field. The coupling between the dilaton and the massless Kaluza-Klein mode of the 3-form is calculated and the coupling constant of the cubic interactions obtained numerically. This coupling are of the order of $\mathrm{Tev}^{-1}$, therefore there exist a possibility to find some signal of it at Tev scale.
\end{abstract}

PACS numbers: 04.60.-m, 04.60.Kz, 03.70.+k

\section{INTRODUCTION}

The core idea of extra dimensions models is to consider the four-dimensional universe as a hyper-surface embedded in multidimensional manifold. The appeal of such models is the determination of scenarios where membranes have the best chances to mimic the standard model's characteristics. In particular, the standard model presents interesting topics to study such as the hierarchy problem, and the cosmological constant problem that can be treated by the above-mentioned models. For example, the Randall-Sundrum model [1, 2] provides a possible solution to the hierarchy problem and show how gravity is trapped to a membrane.

There are studies which concern to general properties of membranes, for example, one that makes an analysis of the space-time singularities that arise in braneworld models with 4D Poincare invariance [3]. Others with cosmological implications, that consider our universe initially as an empty p-brane embedded in a manifold, whose bulk is populated only with a dilaton field that couples itself to the brane, producing a rich and interesting evolutionary cosmology [4]. The presence of other fields in the bulk rises the problem of field localization in the brane, that is an important tool in order to build up the standard model in the membrane setup. For such, several ingredients had to be added: the gravitational field, spinor fields, scalar fields and gauge fields. A lot of aspects have been studied related to these topics. These investigations include both the smooth models described by soliton-like membranes and the pure Randall-Sundrum models containing spacetime singularities. For these there is an extensive list of references in literature [5 16].

Despite its achievements, that model does not favor the localization of zero modes of gauge vector fields. Such localization is important to construct the standard model in a membrane [17]. That happens because the gauge vector field theory is conformal in $D=4$ : this makes the warp factor in the pure Randall-Sundrum model disappear from the effective action. The issue of localization of standard model fields on the three-brane has been widely studied [18 22]. Despite of this, we must consider a scenario in witch it is assumed a priori that the matter fields are constrained to live on the three-brane.

Some studies about higher rank tensor fields have been made showing its relation with the AdS/CFT conjecture [23]. Besides this, String Theory shows the naturalness of higher rank tensor fields in its spectrum [24, 25].

In mathematical terms, the presence of one more extra dimension $(D=5)$ provides the existence of many antisymmetric fields, namely the two, three, four and five forms. However, the only relevant ones for the visible brane are the two and the three form. This is due to the fact that when the number of dimensions increase, also increases the number of gauge freedom. This can be used to cancel the dynamics of the field in the visible brane. The mass spectrum of the two and three form have been studied, for example, in Refs. 26] and [27].

The coupling between the two form and the dilaton, with cosmological consequences, have been studied in 28]. There are also authors that investigate that coupling in domain of standard model physics [29]. Such coupling, inspired in string theory, can provide us with a process that, in principle, could be observed in the LHC. That may happen through a Drell-Yang process, in which a pair of quark-antiquark can give rise to a three form field, mediated by a dilaton. This kind of process can appear in a scenario where the dilaton is considered as the Higgs field 30 32]. This raises the question if the coupling between the three form and the dilaton give a similar process, and this is the goal of this article.

The question to be addressed here in this piece of work 
is related to other kind of gauge fields. We present a full action, including the gravity, the dilaton and the three form field action. The dimensional reduction is done and the effective action at the visible brane is obtained. Then, we analyze carefully the dilaton sector, find the mass spectrum and find the complete solution for dilaton coefficients. This result will be needed when the coupling with the three form is analyzed. After, we analyze the three form sector and, like in the dilaton case, we find the mass spectrum and solve the equation to find the coefficients of the expansion. The mass spectrum of the three form is compared with that of the two form, the dilaton and the gravity field. This gives a hint that the three form must not be seen at LHC, because of the mass of the massive Kaluza-Klein modes. Finally we study the coupling with the dilaton and find how we can conclude that a signal of the massless three form can be found at LHC.

\section{DILATON AND ANTISYMMETRIC TENSOR FIELDS IN RANDALL SUNDRUM FRAMEWORK}

As the number of antisymmetric tensor fields increase with dimensions, these fields has to be considered. In fact these fields have been taken into account in the literature. The fact is that, when the number of dimensions increases, the number of gauge freedom also increases, and this can be used to cancel the degrees of freedom in the visible brane. Therefore the antisymmetric tensors relevant to the visible brane are that of rank two and three [27]. We focus our attention here in the second tensor field cited. As usual, capital Latin index represent the coordinates in the bulk and Greek index, those on the brane. The metric is given by

$$
d s^{2}=e^{-2 \sigma} \eta_{\mu \nu} d x^{\mu} d x^{\nu}+r_{c}^{2} d y^{2}
$$

where $\sigma=k r_{c} y, y$ is the coordinate of fifth dimension, $r_{c}$ is the compactification radius for that dimension, $k$ is a constant of the order of the higher dimensional Planck mass $M$ and $\eta_{\mu \nu}$ is the $4 D$ Minkowski metric.

As said in the introduction, this is a string inspired model. The type IIA superstring posses the two and the three form in the spectrum, the first coming from the NS-NS sector and the other form the RR [25]. The dilaton determines the coupling constant of string theory, therefore the tree level space-time action has an overall factor of it. This means that the coupling of the two form is the same as that of the three form in the effective action. With these arguments at hand, we consider the same coupling as that used recently by Mukhopadhyaya, where a two form is coupled to the dilaton [29]. The action to be considered is therefore given by

$$
S=S_{\text {grav }}+S_{X}+S_{d i l}
$$

where

$$
\begin{gathered}
S_{\text {grav }}=\int d^{5} x \sqrt{-G} 2 M^{3} R \\
S_{X}=\int d^{5} x \sqrt{-G}\left[-e^{\phi / M^{3 / 2}} 2 Y_{M N L P} Y^{M N L P}\right]
\end{gathered}
$$

and

$$
S_{d i l}=\int d^{5} x \sqrt{-G}\left[\frac{1}{2} \partial_{M} \phi \partial^{M} \phi-m^{2} \phi^{2}\right] .
$$

Defining the 3 -form field $X_{M N O}$ and fixing the gauge $X_{\mu \nu y}=0$, we have two possibilities for the strength tensor

$$
Y_{\mu \nu \alpha \beta}=\partial_{[\mu} X_{\nu \alpha \beta]}
$$

or

$$
Y_{y \nu \alpha \beta}=\partial_{[y} X_{\nu \alpha \beta]}
$$

We must here focus only in the dilaton and the antisymmetric field $X_{M N O}$. Thus, for the metric in (1), we have

$$
S_{X}=\int d^{4} x \int d y r_{c} e^{-4 \sigma}\{A+B+C\}
$$

where

$$
\begin{gathered}
A=-2 e^{\phi / M^{3 / 2}} e^{8 \sigma} \eta^{\alpha \beta} \eta^{\mu \nu} \eta^{\lambda \delta} \eta^{\gamma \tau} Y_{\alpha \mu \lambda \gamma} Y_{\beta \nu \delta \tau}, \\
B=-2 e^{\phi / M^{3 / 2}} \frac{4}{r_{c}^{2}} e^{6 \phi} \eta^{\alpha \beta} \eta^{\mu \nu} \eta^{\lambda \gamma} \partial_{y} X_{\alpha \mu \lambda} \partial_{y} X_{\beta \nu \gamma}
\end{gathered}
$$

and

$$
C=r_{c} e^{-4 \sigma}\left[\frac{e^{2 \sigma}}{2} \eta^{\mu \nu} \partial_{\mu} \phi \partial_{\nu} \phi+\frac{1}{2 r_{c}^{2}} \partial_{y} \phi \partial_{y} \phi-m^{2} \phi^{2}\right]
$$

As the mass term of the dilaton decouples the visible brane we must consider the kinetic term only. Performing an integration by parts in the second terms of the dilaton and $X$ fields respectively we get

$$
\begin{aligned}
S & =\int d^{4} x \int d y\left[r_{c} e^{-4 \sigma} A\right. \\
& +e^{\phi / M^{3 / 2}} \frac{8}{r_{c}} \eta^{\alpha \beta} \eta^{\mu \nu} \eta^{\lambda \gamma} X_{\alpha \mu \lambda} \partial_{y}\left(e^{2 \phi} \partial_{y} X_{\beta \nu \gamma}\right) \\
& \left.+\frac{r_{c} e^{-2 \sigma}}{2} \eta^{\mu \nu} \partial_{\mu} \phi \partial_{\nu} \phi-\frac{1}{2 r_{c}} \phi \partial_{y}\left(e^{-4 \sigma} \partial_{y} \phi\right)\right]
\end{aligned}
$$

Note that the derivative in the exponential of the dilaton would give a term suppressed by the Planck mass and therefore is irrelevant for the effective action. At this 
point we must consider the Kaluza-Klein decomposition of the fields

$$
\begin{aligned}
X_{\mu \nu \alpha} & =\sum_{n=0}^{\infty} X_{\mu \nu \alpha}^{n}(x) \frac{\chi^{n}(y)}{\sqrt{r_{c}}} \\
\phi & =\sum_{n=0}^{\infty} \phi^{n}(x) \frac{\psi^{n}(y)}{\sqrt{r_{c}}}
\end{aligned}
$$

where

$$
\begin{gathered}
\int e^{4 \sigma} \chi^{m}(y) \chi^{n}(y) d y=\delta^{m n} \\
\int e^{-2 \sigma} \psi^{m}(y) \psi^{n}(y) d y=\delta^{m n}
\end{gathered}
$$

In terms of the above projections, the effective action on the visible brane is given by

$$
\begin{aligned}
S= & \int d^{4} x \sum_{n=0}^{\infty} \sum_{m=0}^{\infty} \int d y\left\{e^{\phi / M^{3 / 2}}\right. \\
& \times\left[-2 e^{4 \sigma} \chi^{m} \chi^{n} \eta^{\alpha \beta} \eta^{\mu \nu} \eta^{\lambda \delta} \eta^{\gamma \tau} Y_{\alpha \mu \lambda \gamma}^{n} Y_{\beta \nu \delta \tau}^{m}\right. \\
& \left.+\frac{8}{r_{c}^{2}} \eta^{\alpha \beta} \eta^{\mu \nu} \eta^{\lambda \gamma} X_{\alpha \mu \lambda}^{n} X_{\beta \nu \gamma}^{m} \chi^{m} \frac{d}{d y}\left(e^{2 \phi} \frac{d}{d y} \chi^{n}\right)\right] \\
& +\left[\frac{e^{-2 \sigma}}{2} \psi^{m} \psi^{n} \eta^{\mu \nu} \partial_{\mu} \phi^{m} \partial_{\nu} \phi^{n}\right. \\
& \left.\left.-\frac{1}{2 r_{c}^{2}} \phi^{m} \phi^{n} \psi^{m} \partial_{y}\left(e^{-4 \sigma} \partial_{y} \psi^{n}\right)\right]\right\}
\end{aligned}
$$

The action above is the action of the rank three antisymmetric tensor field, or three form, coupled to a dilaton. The first bracket represents the three form coupled to a dilaton and the second the dilaton itself. As there is no coupling of the terms in the second brackets and the three form fields, it is a free dilaton action. So we must concentrate in the free dilaton action and later go on to the main objective, which is the antisymmetric field.

\section{BULK DILATON FIELD}

At this section we must consider the dilaton sector. Here we must expand the dilaton exponential

$$
e^{\phi / M^{3 / 2}}=1+\left(\phi / M^{3 / 2}\right)+\left(\phi / M^{3 / 2}\right)^{2} / 2 !+\ldots
$$

and concentrate only in the first term which will give the usual kinetic and mass term for the dilaton field provided we have

$$
-\frac{1}{r_{c}^{2}} \partial_{y}\left(e^{-4 \sigma} \partial_{y} \psi^{n}\right)=\left(m_{n}^{d}\right)^{2} e^{-2 \sigma} \psi^{n}
$$

The other terms of the expansion will be considered later as interaction terms in the effective action. Using the above orthonormality conditions, we finally get

$$
S_{d i l}=\int d^{4} x \sum_{n=0}^{\infty} \frac{1}{2}\left[\eta^{\mu \nu} \partial_{\mu} \phi^{n} \partial_{\nu} \phi^{n}+\left(m_{n}^{d}\right)^{2} \phi^{n} \phi^{n}\right] .
$$

Therefore we obtain a standard dilaton action with masses given by the solutions of equation (8). The solution is given in 29] and we will basically repeat it here. The easiest solution is for the massless dilaton, where we have to solve the equation

$$
-\frac{1}{r_{c}^{2}} \partial_{y}\left(e^{-4 \sigma} \partial_{y} \psi^{n}\right)=0
$$

with obvious solution

$$
\psi^{0}=\frac{C_{1}}{4 k r_{c}} e^{4 \sigma}+C_{2} .
$$

The constants can be obtained from orbifold condition $\psi^{0}(-\pi)=\psi^{0}(\pi)$ and from orthonormality conditions, again. We find $C_{1}=0$ and, considering also that $\exp \left(-2 k r_{c} \pi\right)<<1, C_{2}=\sqrt{k r_{c}}$. The final solution is

$$
\psi^{0}=\sqrt{k r_{c}}
$$

In order to solve the equations for the massive modes first we must make the change of variables $z_{n}=\frac{m_{n}^{d}}{k} e^{\sigma}$ and $f^{n}=\psi^{n} e^{-2 \sigma}$ to obtain the second order equation

$$
\left[z_{n}^{2} \frac{d^{2}}{d z_{n}^{2}}+z_{n} \frac{d}{d z_{n}}+\left(z_{n}^{2}-4\right)\right] f_{n}=0
$$

which admits a Bessel function of order 2 as a solution. The general solution is

$$
\psi^{n}=\frac{e^{2 \sigma}}{N_{n}}\left[J_{2}\left(z_{n}\right)+\alpha_{n} Y_{2}\left(z_{n}\right)\right]
$$

where $N_{n}$ and $\alpha_{n}$ are constants to be determined. First of all we must to use the continuity condition for the derivative of $\psi^{n}$ at $y=0$. Remembering that $J_{2}$ and $Y_{2}$ are Bessel and Neumann functions of order 2 we obtain

$$
\alpha_{n}=-\frac{J_{1}\left(\frac{m_{n}^{d}}{k}\right)}{Y_{1}\left(\frac{m_{n}^{d}}{k}\right)}
$$

As the masses $m_{n}^{d}$ are expected to be of order of Tev on the brane we have $m_{n}^{d}<<k$ and expanding the rhs of the above equation we obtain

$$
\alpha_{n}=-\frac{\pi}{4}\left(\frac{m_{n}^{d}}{k}\right)^{2}<<1 .
$$

Using the boundary condition at $y=\pi$ we obtain

$$
J_{1}\left(x_{n}\right)=0
$$

where we have defined $x_{n}=z_{n}(\pi)=\frac{m_{n}^{d}}{k} e^{k r_{c} \pi}$.

If we find the solutions of the equation above we can find $x_{n}$ and therefore the mass spectrum. Now the orthogonality condition can be used to determine $N_{n}$ and we obtain for the solution

$$
\psi^{n}\left(z_{n}\right)=\sqrt{k r_{c}} \frac{e^{2 \sigma}}{e^{k r_{c} \pi}} \frac{J_{2}\left(z_{n}\right)}{J_{2}\left(x_{n}\right)} .
$$


Now we have the complete solution for the dilaton given by

$$
\phi=\sqrt{k r_{c}} \phi^{0}+\sum_{n=1}^{\infty} \sqrt{k} \frac{e^{2 \sigma}}{e^{k r_{c} \pi}} \frac{J_{2}\left(z_{n}\right)}{J_{2}\left(x_{n}\right)} \phi^{n}
$$

with masses given by equation (9). Posteriorly we will compare the mass of the dilaton with the graviton one. The complete solution for the dilaton will be necessary for studying its coupling to the antisymmetric field.

\section{BULK THREE FORM FIELD}

Now we must consider the three form field. The action was constructed before and we have

$$
\begin{aligned}
S_{X} & =\int d^{4} x \sum_{n=0}^{\infty} \sum_{m=0}^{\infty} \int d y e^{\phi / M^{3 / 2}} \\
& {\left[-2 e^{4 \sigma} \chi^{m} \chi^{n} \eta^{\alpha \beta} \eta^{\mu \nu} \eta^{\lambda \delta} \eta^{\gamma \tau} Y_{\alpha \mu \lambda \gamma}^{n} Y_{\beta \nu \delta \tau}^{m}\right.} \\
& \left.+\frac{8}{r_{c}^{2}} \eta^{\alpha \beta} \eta^{\mu \nu} \eta^{\lambda \gamma} X_{\alpha \mu \lambda}^{n} X_{\beta \nu \gamma}^{m} \chi^{m} \frac{d}{d y}\left(e^{2 \phi} \frac{d}{d y} \chi^{n}\right)\right]
\end{aligned}
$$

Again we must expand of the dilaton exponential and consider only the first term. The mass spectrum is obtained from

$$
-\frac{1}{r_{c}^{2}} \frac{d}{d y}\left(e^{2 \phi} \frac{d}{d y} \chi^{n}\right)=\left(m_{X}^{n}\right)^{2} \chi^{n} e^{4 \sigma}
$$

and again the other terms of the expansion will be considered later as interaction terms in the effective action. The simplest and most important solution of the above equation is obtained for the massless case, where we have the equation

$$
-\frac{1}{r_{c}^{2}} \frac{d}{d y}\left(e^{2 \phi} \frac{d}{d y} \chi^{n}\right)=0
$$

with obvious solution

$$
\chi^{0}=-\frac{C_{1}}{2 k r_{c}} e^{-2 \sigma}+C_{2}
$$

Using continuity of the derivative at $y=\pi$ give us $C_{1}=0$ and from orthogonality we finally get

$$
\chi^{0}=\sqrt{2 k r_{c}} e^{-2 k r_{c} \pi}
$$

For the massive modes, similarly to the dilaton case, we perform the redefinitions $z_{n}^{\prime}=\frac{m_{n}}{k} e^{\sigma}$ and $f_{n}^{\prime}=e^{\sigma} \chi^{n}$ to obtain the equation

$$
\left[z_{n}^{\prime 2} \frac{d^{2}}{d z_{n}^{\prime 2}}+z_{n}^{\prime} \frac{d}{d z_{n}^{\prime}}+\left(z_{n}^{\prime 2}-1\right)\right] f_{n}^{\prime}=0
$$

with solution given by a Bessel function of order 1 . Therefore

$$
\chi^{n}=e^{-\sigma} f_{n}^{\prime}=\frac{e^{-\sigma}}{N_{n}^{\prime}}\left[J_{1}\left(z_{n}^{\prime}\right)+\alpha_{n}^{\prime} Y_{1}\left(z_{n}^{\prime}\right)\right],
$$

and again we have to determine the constants $N_{n}^{\prime}$ and $\alpha_{n}^{\prime}$ using contour conditions. First of all we must use continuity conditions at $y=0$ to obtain

$$
\alpha_{n}^{\prime}=-\frac{J_{2}\left(\frac{m_{X}^{n}}{k}\right)}{Y_{2}\left(\frac{m_{X}^{n}}{k}\right)} .
$$

As the masses are in Tev scale we have $m_{X}^{n}<<k$ and expanding the above expression we get

$$
\alpha_{n}^{\prime} \sim \frac{\pi}{2^{5}}\left(\frac{m_{X}^{n}}{k}\right)^{4}<<1 .
$$

Using the above result, the fact that $e^{k r_{c} \pi}>>1$ and the contour condition at $y=\pi$ we obtain

$$
J_{2}\left(x_{n}^{\prime}\right)=0
$$

with the definition $x_{n}^{\prime}=z_{n}^{\prime}(\pi)=\frac{m_{X}^{n}}{k} e^{k r_{c} \pi}$. Therefore we can obtain the mass spectrum and, in the visible brane, the masses are solutions of the equation (11). The effective action is given by

$$
\begin{aligned}
S_{X} & =\int d^{4} x \sum_{n=0}^{\infty} e^{\phi / M^{3 / 2}}\left\{-2 \eta^{\alpha \beta} \eta^{\mu \nu} \eta^{\lambda \delta} \eta^{\gamma \tau} Y_{\alpha \mu \lambda \gamma}^{n} Y_{\beta \nu \delta \tau}^{m}\right. \\
& \left.-8\left(m_{X}^{n}\right)^{2} \eta^{\alpha \beta} \eta^{\mu \nu} \eta^{\lambda \gamma} X_{\alpha \mu \lambda}^{n} X_{\beta \nu \gamma}^{m}\right\}
\end{aligned}
$$

We can obtain $N_{n}$ with the normalization condition to obtain

$$
N_{n}^{\prime}=\frac{e^{k r_{c} \pi}}{\sqrt{k r_{c}}} J_{1}\left(x_{n}^{\prime}\right)
$$

and we arrive at the final solution for the massive modes given by

$$
\chi^{n}\left(z_{n}\right)=\sqrt{k r_{c}} \frac{e^{\sigma}}{e^{k r_{c} \pi}} \frac{J_{1}\left(z_{n}^{\prime}\right)}{J_{1}\left(x_{n}^{\prime}\right)} .
$$

It is interesting to note that, despite the way we have defined it, the mass spectrum of the three form field is no altered by the dilaton, and the only change will be in the interaction terms, that will be analyzed posteriorly. We list in table $\llbracket$ the value of the masses for the graviton, the dilaton, the two and three form, as per [27, 29], using the scale $k r_{c}=12$ and $k=10^{19} \mathrm{Gev}$

\begin{tabular}{|c|cccc|}
\hline$n$ & 1 & 2 & 3 & 4 \\
\hline$m_{\text {grav }}^{n}(\mathrm{TeV})$ & 1.66 & 3.04 & 4.40 & 5.77 \\
\hline$m_{\text {dil }}^{n}(\mathrm{TeV})$ & 1.66 & 3.04 & 4.40 & 5.77 \\
\hline$m_{K R}^{n}(\mathrm{TeV})$ & 2.87 & 5.26 & 7.62 & 9.99 \\
\hline$m_{X}^{n}(\mathrm{TeV})$ & 4.44 & 7.28 & 10.05 & 12.79 \\
\hline
\end{tabular}

TABLE I: Masses of KK modes where $k r_{c}=12$ and $k=$ $10^{19}$ Gev 
It is obvious from the table प that, higher is the rank of the tensor, higher is the mass spectrum, and the possibility of a finding these field in the LHC becomes more and more elusive. The possibility for some signal of the $X$ field in LHC will comes from its interaction with the dilaton field and this is analyzed in the next section.

\section{THREE FORM COUPLED TO DILATON}

As we are going to explore here only the coupling between the three form and the dilaton, we must consider only the relevant part of the action, which is given by

$$
\begin{aligned}
S_{X} & =\int d^{4} x \sum_{n=0}^{\infty} \sum_{m=0}^{\infty} \int d y e^{\phi / M^{3 / 2}} \\
& \times\left[-2 e^{4 \sigma} \chi^{m} \chi^{n} \eta^{\alpha \beta} \eta^{\mu \nu} \eta^{\lambda \delta} \eta^{\gamma \tau} Y_{\alpha \mu \lambda \gamma}^{n} Y_{\beta \nu \delta \tau}^{m}\right. \\
& \left.-8\left(m_{X}^{n}\right)^{2} \eta^{\alpha \beta} \eta^{\mu \nu} \eta^{\lambda \gamma} X_{\alpha \mu \lambda}^{n} X_{\beta \nu \gamma}^{m} \chi^{m} \chi^{n} e^{4 \sigma}\right] .
\end{aligned}
$$

When we expand the exponential of the dilaton, the first term will give us the usual kinetic and mass term studied previously. The next terms of the expansion are

$$
\begin{aligned}
\exp \left(\phi / M^{3 / 2}\right)-1 & =\left(M^{-3 / 2} \sum_{n=0}^{\infty} \phi^{n}(x) \frac{\psi^{n}(y)}{\sqrt{r_{c}}}\right) \\
& +\frac{1}{2 !}\left(M^{-3 / 2} \sum_{n=0}^{\infty} \phi^{n}(x) \frac{\psi^{n}(y)}{\sqrt{r_{c}}}\right)^{2}+\ldots
\end{aligned}
$$

The terms beyond the first are highly suppressed by powers of the plank mass, therefore we must consider only the first term, or

$$
\begin{aligned}
& \exp \left(\phi / M^{3 / 2}\right)-1 \sim M^{-3 / 2} \sum_{n=0}^{\infty} \phi^{n}(x) \frac{\psi^{n}(y)}{\sqrt{r_{c}}} \\
& =M^{-3 / 2}\left(\sqrt{k r_{c}} \phi^{0}+\sum_{n=1}^{\infty} \sqrt{k} \frac{e^{2 \sigma}}{e^{k r_{c} \pi}} \frac{J_{2}\left(z_{n}\right)}{J_{2}\left(x_{n}\right)} \phi^{n}\right) .
\end{aligned}
$$

The above term will give a coupling of the form $\phi X^{2}$. As we can see from the complete solution of the dilaton and the three form, the interaction of the massless fields will be suppressed by a factor of $\frac{1}{M_{p}}$ and therefore is not considered. The interaction between a massless dilaton, a massless two form and a massive three form is like $\phi^{0} X^{0} X^{n}$ and give us a null result because of the orthogonality relations.

Therefore the interaction that posses massless dilatons are irrelevant, and we must consider only the massive ones. Taking in account these considerations and rearranging terms in the action, we get for the cubic interac- tions

$$
\begin{aligned}
S_{X} & \supset S_{c u b i c}=\sum_{n, m=0}^{\infty} \sum_{p=1}^{\infty} \frac{M^{-3 / 2}}{\sqrt{r_{c}}} \int d y e^{4 \sigma} \chi^{m}(y) \chi^{n}(y) \psi^{p}(y) \\
& \times \int d^{4} x \phi^{p}(x)\left[-2 \eta^{\alpha \beta} \eta^{\mu \nu} \eta^{\lambda \delta} \eta^{\gamma \tau} Y_{\alpha \mu \lambda \gamma}^{n} Y_{\beta \nu \delta \tau}^{m}\right. \\
& \left.-8\left(m_{X}^{n}\right)^{2} \eta^{\alpha \beta} \eta^{\mu \nu} \eta^{\lambda \gamma} X_{\alpha \mu \lambda}^{n} X_{\beta \nu \gamma}^{m}\right] .
\end{aligned}
$$

From the effective action we can see that the coupling constant for these interactions are given by

$$
\frac{M^{-3 / 2}}{\sqrt{r_{c}}} \int_{-\pi}^{+\pi} d y e^{4 \sigma} \chi^{m} \chi^{n} \psi^{i}
$$

As said before, due the large values of the mass for the KK modes, their signal must hardly be seen at LHC. Therefore we concentrate in the more interesting case, that is the massless mode. Using now the solution for the coefficients above we obtain, for the massless modes

$$
\frac{2 k r_{c}}{M_{P}} e^{-5 k r_{c} \pi} \int_{-\pi}^{+\pi} d y e^{6 \sigma} \frac{J_{2}\left(z_{n}\right)}{J_{2}\left(x_{n}\right)}
$$

The above integral was solved numerically using the software Mathematica, and the results for the first four KK modes are given in table

\begin{tabular}{|c|c|}
\hline$n$ & Coupling constant \\
\hline 1 & 0.00143676 \\
\hline 2 & 0.000788648 \\
\hline 3 & 0.000413211 \\
\hline 4 & 0.000249329 \\
\hline
\end{tabular}

TABLE II: Coupling constants in units of $G e v^{-1}$

The values of the coupling constant in the above table are given in $\mathrm{Gev}^{-1}$. This show a rather interesting possibility: Despite the fact that the massless mode is extremely suppressed, its coupling with the dilaton rises the possibility of a signal at LHC. A pointed in 29], the Lorenz structure of the interactions and masses involved will change the angular distribution of $\mathrm{X}$ fields produced. Furthermore, in the a Drell-Yan process, the production of $\mathrm{X}$ zero modes through, for example, the interaction of quark-antiquark pairs, may have different (but significant) rates for sufficient integrated luminosity if we compare with the case of the Kalb-Ramond and gravity fields.

\section{CONCLUSIONS}

Extending earlier results about antisymmetric fields 27] we have shown here that the non-trivial coupling between the dilaton and the antisymmetric field don't 
affect its high suppression in our visible brane due to the warp factor of the Randall-Sundrum scenario.

In the dilaton background we have studied new interactions between the Kaluza-Klein modes of the dilaton field with the antisymmetric field. These results are important from the phenomenological viewpoint. The interactions that posses massless dilatons are irrelevant, and we must consider only the massive ones. As we have shown, higher order interactions of the massless fields are suppressed by a factor of $\frac{1}{M_{p}}$ and therefore are not considered. Due to the large mass of the massive modes, we considered only the massless case. In fact, numerical computation of the important interaction is of order of $\mathrm{Tev}^{-1}$ and can give us signals of this field in the LHC's searches through DrellYan processes mediated by massive dilaton.

We thank Biswarup Mukhopadhyaya for helpful comments. The authors would like to acknowledge the financial support provided by Fundação Cearense de Apoio ao Desenvolvimento Científico e Tecnológico (FUNCAP) and the Conselho Nacional de Desenvolvimento Científico e Tecnológico (CNPq).

This paper is dedicated to the memory of my wife Isabel Mara (R. R. Landim)

[1] L. Randall and R. Sundrum, "An alternative to compactification," Phys. Rev. Lett. 83, 4690 (1999) arXiv:hep-th/9906064.

[2] L. Randall and R. Sundrum, "A large mass hierarchy from a small extra dimension," Phys. Rev. Lett. 83, 3370 (1999) arXiv:hep-ph/9905221.

[3] C. A. Herrera-Aguilar et al., "Aspects of thick brane worlds: 4D gravity localization, smoothness, and mass gap," Presented at 1st Mediterranean Conference in Classical and Quantum Gravity, Kolymbari, Crete, Greece, 14-18 Sep 2009. [arXiv:hep-th/0910.0363].

[4] G. De Risi, "Massless and massive graviton spectra in anisotropic dilatonic braneworld cosmologies," Phys. Rev. D 73, 124015 (2006) arXiv:gr-qc/0601132.

[5] J. Liang and Y. S. Duan, "Localization Of Matters On The Bent Brane In Ads5 Bulk," Phys. Lett. B 680, 489 (2009).

[6] J. Liang and Y. S. Duan, "Localization and mass spectrum of spin-1/2 fermionic field on a thick brane with Poincare symmetry," Europhys. Lett. 87, 40005 (2009).

[7] Y. X. Liu, H. Guo, C. E. Fu and J. R. Ren, "Localization of Matters on Anti-de Sitter Thick Branes," JHEP 1002, 080 (2010) arXiv:0907.4424 [hep-th]].

[8] A. Flachi and M. Minamitsuji, "Field localization on a brane intersection in anti-de Sitter spacetime," Phys. Rev. D 79, 104021 (2009) arXiv:0903.0133 [hep-th]].

[9] R. Koley, J. Mitra and S. SenGupta, "Fermion localization in generalised Randall Sundrum model," Phys. Rev. D 79, 041902 (2009) arXiv:0806.0455 [hep-th]].

[10] D. Bazeia, A. R. Gomes and L. Losano, "Gravity localization on thick branes: a numerical approach," Int. J. Mod. Phys. A 24, 1135 (2009) [arXiv:0708.3530 [hep-th]].

[11] Y. X. Liu, X. H. Zhang, L. D. Zhang and Y. S. Duan,
"Localization of Matters on Pure Geometrical Thick Branes," JHEP 0802, 067 (2008) arXiv:0708.0065 [hepth]].

[12] N. Barbosa-Cendejas and A. Herrera-Aguilar, "Localization of $4 \mathrm{D}$ gravity on pure geometrical Thick branes," Phys. Rev. D 73, 084022 (2006) [Erratum-ibid. D 77, 049901 (2008)] arXiv:hep-th/0603184.

[13] P. Midodashvili and L. Midodashvili, "Gravitational localization of matters in 6D," Europhys. Lett. 65, 640 (2004) arXiv:hep-th/0308039.

[14] A. Kehagias and K. Tamvakis, "Localized gravitons, gauge bosons and chiral fermions in smooth spaces generated by a bounce," Phys. Lett. B 504, 38 (2001) arXiv:hep-th/0010112.

[15] Y. X. Liu, C. E. Fu, H. Guo, S. W. Wei and Z. H. Zhao, "Bulk Matters on a GRS-Inspired Braneworld," arXiv:1002.2130 [hep-th].

[16] R. Davies and D. P. George, "Fermions, scalars and Randall-Sundrum gravity on domain-wall branes," Phys. Rev. D 76, 104010 (2007) arXiv:0705.1391 [hep-ph]].

[17] H. Davoudiasl, J. L. Hewett and T. G. Rizzo, "Bulk gauge fields in the Randall-Sundrum model," Phys. Lett. B 473, 43 (2000) arXiv:hep-ph/9911262.

[18] V. A. Rubakov and M. E. Shaposhnikov, "Do We Live Inside A Domain Wall?," Phys. Lett. B 125, 136 (1983).

[19] G. R. Dvali and M. A. Shifman, "Domain walls in strongly coupled theories," Phys. Lett. B 396, 64 (1997) [Erratum-ibid. B 407, 452 (1997)] arXiv:hep-th/9612128.

[20] A. Pomarol, "Gauge bosons in a five-dimensional theory with localized gravity," Phys. Lett. B 486, 153 (2000) arXiv:hep-ph/9911294.

[21] Y. Grossman and M. Neubert, "Neutrino masses and mixings in non-factorizable geometry," Phys. Lett. B 474, 361 (2000) arXiv:hep-ph/9912408.

[22] N. Arkani-Hamed, L. J. Hall, D. Tucker-Smith and N. Weiner, "Solving the hierarchy problem with exponentially large dimensions," Phys. Rev. D 62, 105002 (2000) arXiv:hep-ph/9912453.

[23] C. Germani and A. Kehagias, "Higher-spin fields in braneworlds," Nucl. Phys. B 725, 15 (2005) arXiv:hep-th/0411269.

[24] J. Polchinski, "String theory. Vol. 1: An introduction to the bosonic string," SPIRES entry Cambridge, UK: Univ. Pr. (1998) 402 $p$

[25] J. Polchinski, "String theory. Vol. 2: Superstring theory and beyond," SPIRES entry Cambridge, UK: Univ. Pr. (1998) $531 p$

[26] B. Mukhopadhyaya, S. Sen, S. Sen and S. SenGupta, "Bulk Kalb-Ramond field in Randall Sundrum scenario," Phys. Rev. D 70, 066009 (2004) arXiv:hep-th/0403098

[27] B. Mukhopadhyaya, S. Sen and S. SenGupta, "Bulk antisymmetric tensor fields in a Randall-Sundrum model," Phys. Rev. D 76, 121501 (2007) arXiv:0709.3428 [hepth]].

[28] G. De Risi, "Bouncing cosmology from Kalb-Ramond Braneworld," Phys. Rev. D 77, 044030 (2008) arXiv:0711.3781 [hep-th]].

[29] B. Mukhopadhyaya, S. Sen and S. SenGupta, "A Randall-Sundrum scenario with bulk dilaton and torsion," Phys. Rev. D 79, 124029 (2009) arXiv:0903.0722 [hep-th]].

[30] M. G. Ryskin and A. G. Shuvaev, "Higgs Boson as a Dilaton," Phys. Atom. Nucl. 73, 965 (2010) arXiv:0909.3374 
[hep-ph]].

[31] R. Foot, A. Kobakhidze and K. L. McDonald, "Dilaton as the Higgs boson," arXiv:0812.1604 [hep-ph].

[32] W. D. Goldberger, B. Grinstein and W. Skiba, "Light scalar at LHC: the Higgs or the dilaton?," Phys. Rev. Lett. 100, 111802 (2008) arXiv:0708.1463 [hep-ph]]. 